\title{
BMJ Open Patient engagement, treatment preferences and shared decision-making in the treatment of opioid use disorder in adults: a scoping review protocol
}

\author{
Tyler Marshall,, ${ }^{1}$ Elizabeth N Kinnard, ${ }^{2}$ Myles Hancock, ${ }^{3}$ Susanne King-Jones, ${ }^{4}$ \\ Karin Olson, ${ }^{5}$ Adam Abba-Aji, ${ }^{1}$ Katherine Rittenbach, ${ }^{6}$ Sunita Vohra ${ }^{1,4,7}$
}

To cite: Marshall T, Kinnard EN, Hancock M, et al. Patient engagement, treatment preferences and shared decision-making in the treatment of opioid use disorder in adults: a scoping review protocol. BMJ Open 2018;8:e022267. doi:10.1136/ bmjopen-2018-022267

- Prepublication history for this paper is available online. To view these files, please visit the journal online (http://dx.doi. org/10.1136/bmjopen-2018022267).

Received 9 February 2018 Revised 18 July 2018 Accepted 27 August 2018

Check for updates

(C) Author(s) (or their employer(s)) 2018. Re-use permitted under CC BY-NC. No commercial re-use. See rights and permissions. Published by BMJ.

For numbered affiliations see end of article.

Correspondence to

Dr Sunita Vohra;

svohra@ualberta.ca

\section{ABSTRACT}

Introduction Opioid use disorder (OUD) is characterised by the fifth Edition of the Diagnostic and Statistics Manual as a problematic pattern of opioid use (eg, fentanyl, heroin, oxycodone) that leads to clinically significant impairment. OUD diagnoses have risen substantially over the last decade, and treatment services have struggled to meet the demand. Evidence suggests when patients with chronic illnesses are matched with their treatment preferences and engaged in shared decision-making (SDM), health outcomes may improve. However, it is not known whether SDM could impact outcomes in specific substance use disorders such as OUD.

Methods and analysis A scoping review will be conducted according to Arksey and O'Malley's framework and by recommendations from Levac et al. The search strategy was developed to retrieve relevant publications from database inception and June 2017. MEDLINE, EMBASE, PsycINFO, Cochrane Database for Controlled Trials, Cochrane Database for Systematic Reviews and reference lists of relevant articles and Google Scholar will be searched. Included studies must be composed of adults with a diagnosis of OUD, and investigate SDM or its constituent components. Experimental, quasi-experimental, qualitative, case-control, cohort studies and cross-sectional surveys will be included. Articles will be screened for final eligibility according to title and abstract, and then by full text. Two independent reviewers will screen excluded articles at each stage. A consultation phase with expert clinicians and policy-makers will be added to set the scope of the work, refine research questions, review the search strategy and identify additional relevant literature. Results will summarise whether SDM impacts health and patient-centred outcomes in OUD.

Ethics and dissemination Scoping review methodology is considered secondary analysis and does not require ethics approval. The final review will be submitted to a peer-reviewed journal, disseminated at relevant academic conferences and will be shared with policy-makers, patients and clinicians.

\section{INTRODUCTION}

\section{Shared decision-making}

Shared decision-making (SDM) has been considered by many medical experts to be an
Strengths and limitations of this study

- This will be the first scoping review to investigate shared decision-making, treatment preferences and patient engagement in the treatment of a specific, substance use disorder such as opioid use disorder (OUD).

- Our large multidisciplinary research team will perform a comprehensive search spanning five electronic databases, in addition to a hand-search of reference lists and Google Scholar.

- Two reviewers will independently screen and extract articles for inclusion, and quality of included studies will be assessed.

- Public health researchers, OUD experts and policy-makers will be consulted throughout the study to collect additional literature, enhance methodological rigour and promote clinical and policy relevance.

- For feasibility constraints, this review will not be able to search for articles other than in English language.

integral approach for achieving patient-centred care in clinical medicine. ${ }^{12}$ Elwyn $e t a l^{2}$ define SDM as 'an approach where clinicians and patients share the best available evidence when faced with the task of making decisions, and where patients are supported to consider options to achieve informed preferences.' Furthermore, there is evidence to suggest that informing patients of treatment options and including their preferences into the decision-making process may promote favourable health outcomes. ${ }^{2-4}$ SDM is particularly well supported within the context of primary care and chronic disease management. ${ }^{5}$ SDM demonstrated increased positive outcomes such as patient satisfaction, treatment adherence and engagement in many chronic conditions such as diabetes. ${ }^{45}$ However, the specific impact of SDM on health outcomes in mental health disorders, such as opioid use disorder (OUD), remains unclear. Further research in 
this area has been recommended by Friedrichs $e t a l,{ }^{6}$ policy-makers and expert clinicians.

\section{The opioid crisis and OUD}

The US Centers for Disease Control and Prevention (CDC) and Health Canada declared a 'public health crisis' in response to sharply rising opioid-related morbidity and mortality since $2000 .^{78}$ During this time period, opioid-related overdose morbidity and mortality have increased nearly fourfold. ${ }^{8-10}$ Some experts suggest that opioid-related mortality data may be underestimated, as nearly $25 \%$ of drug-related death certificates do not report what particular substances were involved. ${ }^{10}$ Since around 2010, there has been a surge of synthetic opioid use (eg, fentanyl, carfentanil), ${ }^{11}$ which is associated with increased morbidity and mortality rates of over $200 \%$ in the USA and Canada. ${ }^{7812}$ These potent opioids are predominately illicitly manufactured, ${ }^{11}$ and are now primarily driving the increased rate of fatal drug overdoses across North America. ${ }^{12-14}$ Subsequently, mortality rates associated with diverted prescription opioids have begun to plateau and decrease in many areas. ${ }^{12} 13$

OUD is characterised by the fifth Edition of the Diagnostics and Statistics Manual (DSM-V) as a "problematic pattern of opioid use leading to clinically significant impairment or distress' over a 12-month period. ${ }^{15}$ Addiction experts now most often refer to OUD as a 'biopsychosocial' disorder. ${ }^{16}{ }^{17}$ Increased prevalence of OUD is associated with increased wait times for inpatient and outpatient mental health and addiction services in many parts of North America. ${ }^{18-20}$ As a result, community-based mental health and primary care facilities have been confronted with an increased need for evidence-based OUD treatments such as opioid agonist treatment (OAT) (eg, methadone, buprenorphine/naloxone) $),{ }^{19} 21$ in addition to harm reduction services (eg, providing naloxone kits), and drug user education services. ${ }^{22} 23$ Although regulations have been changing rapidly, not all clinicians can prescribe OAT, which limits access to treatment for many individuals with OUD, ${ }^{24}$ particularly in rural areas. ${ }^{25}$ Further, issues such as lack of insurance coverage for $\mathrm{OAT}^{26}$ and high rates of relapse ${ }^{27}$ may imply that current treatment options are neither available nor sufficient for many people with OUD. However, since involving patients in SDM has shown to improve outcomes in some chronic diseases, we think it is reasonable to explore whether SDM impacts various health and patient-centred outcomes for adults with OUD.

\section{Rationale for review}

We believe carrying out this scoping review helps address a critical need in current public healthcare. Opioid-related morbidity and mortality have increased more than fourfold since 1999 and continues to rise. ${ }^{818}$ As a result, national health regulatory bodies in Canada and the USA have declared an 'opioid crisis,' requiring immediate attention and innovative treatment solutions to reduce the prevalence of OUD. ${ }^{89} 18$ Patients with OUD often experience long wait times to receive treatment ${ }^{20}$ due to rapidly increasing OUD prevalence rates, ${ }^{12829}$ and high incidence rates of relapse postdischarge ${ }^{27}$ Furthermore, poor adherence or non-compliance to OAT postdischarge yields an increased risk of relapse and overdose due to a rapid decrease in opioid tolerance. $^{30}$

There is additional evidence suggesting that patient attitudes and beliefs regarding a particular treatment may impact treatment entry and adherence in patients with OUD. ${ }^{31}$ Further, increased therapeutic alliance has shown to be a predictor for improvement in various substance use treatment outcomes (eg, treatment adherence, patient engagement). ${ }^{32}$ SDM has been found to improve patient outcomes when incorporated into the treatment strategy in chronic diseases, ${ }^{4}$ but has not been fully explored in the patient population with OUD. Experts have suggested that implementing patient-centred care, reducing stigma around substance use, and improving treatment adherence are major goals for treating OUD and preventing overdose ${ }^{33-37}$ As a result, we state it is justifiable to assess the depth and breadth of the evidence of SDM within the context of OUD treatment in order to guide future research and care in this field.

In 2016, a systematic review by Friedrichs et at was conducted reviewing treatment options, patient preferences and SDM for the treatment of various substance use disorders (eg, alcohol use disorder). Based on the 25 included articles, the authors suggested that SDM be implemented in the treatment of substance use disorders; however, Friedrichs et al also stated that definitive conclusions regarding the effectiveness of SDM for various specific substance use disorders could not yet be established due to the heterogeneity of the included studies. ${ }^{6}$ In this systematic review, six articles related to OUD were identified, and only one was of experimental design. ${ }^{6}$ Moreover, we believe that our proposed scoping review may be able to further contribute to the existing literature by employing a search strategy includes more opioid-related search terms, while focusing the analysis on understanding if incorporating SDM into the OUD treatment approach has any impact on adults with OUD.

\section{Objectives of the review}

The objectives for this scoping review are to (1) summarise the impact of SDM on health-related and patient-centred outcomes for adults with OUD and (2) establish the breadth and depth of the relevant scientific literature. This will be the first study to systematically review SDM and its related constructs, such as patient engagement and patient preferences within the context of OUD. As of 2013, the DSM-V categorises 'opioid dependence' and 'opioid addiction' under OUD ${ }^{38}$; ; although, there are distinctions between the two respective conditions. ${ }^{38}$ As a result, both of these terms will be searched for exclusively in adults outside the context of treatment for chronic, cancer-related pain. 


\section{METHODS AND ANALYSIS}

\section{Stage 1: identifying the research questions}

Scoping reviews answer broad research questions intended to map the current state of the literature and identify gaps in research or current understanding. ${ }^{40-43}$ SDM for the treatment of specific substance use disorders such as OUD may benefit from such an approach. Additionally, there is promising evidence suggesting that incorporating patient preferences into chronic disease treatment plans in primary care settings may improve other important outcomes such as patient satisfaction, engagement, knowledge gain and/or treatment adherence. ${ }^{24}$ For this scoping review, SDM will be defined consistently with the definition provided by Elwyn et al, who defined SDM as 'an approach where clinicians and patients are supported to consider options to achieve informed preferences. ${ }^{2}$ Since SDM is often viewed as a 'general' approach to care, ${ }^{44}$ and may not always be controlled or delivered ubiquitously, we decided a broad research question would be best to suit a review on this topic.

We chose adults (18 years of age or older) to be the target population because they are most commonly legally able to participate in medical decisions without parental support. ${ }^{45}$ As result, we believe exploring SDM within the context of OUD treatment in adults is worthwhile. Therefore, this review will assess the most relevant literature, identify gaps in knowledge and explore fundamental concepts in regard to SDM and the treatment of OUD in adults.

The research question for this review is: What evidence exists regarding the use of SDM and related elements for improving health-related and patient-centred outcomes in adults with OUD?

\section{Stage 2: identifying relevant studies \\ Search strategy}

Our multidisciplinary team developed a comprehensive preliminary search strategy and study selection framework, in consultation with expert stakeholders and a health research librarian. We consulted other systematic reviews ${ }^{4646}$ on SDM and developed a search strategy using free-text search terms and Medical Subject Headings related to OUD and SDM. We best understand SDM as a broad construct that includes concepts such as 'patient participation', 'patient preferences', 'patient engagement', 'patient autonomy', 'decision-making', 'self-care', 'decision support', 'consumer engagement' and 'consumer participation'. As a result, we included all of these terms in our search strategy. Due to feasibility restraints, the search was limited to English language articles, and there was no restriction on publication year. We subsequently performed a preliminary prescreen of MEDLINE and retrieved 237 articles (box 1).

EMBASE, PsycINFO, Cochrane Central Register of Controlled Trials and Cochrane Database of Systematic Reviews, reference lists and Google Scholar will also be searched for relevant studies throughout the review
Box 1 Preliminary MEDLINE search strategy

1. (opi* adj2 addiction).mp.

2. exp Opioid-Related Disorders/

3. (opi* adj2 misuse).mp.

4. opi* abuse.mp.

5. $\left(\right.$ opi* $^{\star}$ adj1 depen $\left.{ }^{\star}\right) \cdot m p$.

6. exp Opiate Substitution Treatment/

7. Opioid-Related Disorder*.ti,ab.

8. Opioid disorder.ti,ab.

9. or $/ 1-8$

10. Patient Preference/

11. patient preference.ti,ab.

12. Patient Participation/

13. patient participation.ti,ab.

14. patient involvement.ti,ab.

15. patient engagement.ti,ab.

16. patient perspective.ti,ab.

17. consumer participation.ti,ab.

18. consumer engagement.ti,ab.

19. Decision Making/

20. shared decision making.ti,ab.

21. patient autonomy.ti,ab.

22. decision support.ti,ab.

23. (patient centered or patient centred).ti,ab.

24. Patient-Centered Care/

25. or $/ 10-24$

26. 9 and 25

27. limit 26 to english language

process. Experts will be contacted for their input and asked for any additional literature until no new relevant articles are found. After a saturation point is reached, duplicates will be removed, and the retrieved studies will be assessed for inclusion.

\section{Stage 3: study selection}

Eligibility criteria

The Population, Intervention, Comparator, Outcome, Study design model will be used to develop an eligibility criteria framework (table 1).

\section{Inclusion criteria}

This review will include studies only with adults (mean age 18 years or older) who have been diagnosed with OUD (DSM-V), opioid dependence or opioid addiction (DSM-IV and prior). Studies that involve 'heroin users' will also be included. Studies where patients were mandated to treatment (eg, drug court mandate) will be excluded, in addition to studies of alcohol or other substance use disorders. Studies then must either (1) directly study SDM via observation or SDM intervention (eg, SDM tool), (2) study patient preferences or comparable construct (eg, consumer preference) or (3) study patient engagement or comparable construct (eg, consumer engagement).

The following study types will be included for review: experimental studies (randomised controlled trials), quasi-experimental studies (controlled designs without randomisation), systematic reviews, observational studies 


\begin{tabular}{|c|c|c|}
\hline PICOS & Included & Excluded \\
\hline Population & $\begin{array}{l}\text { Studies with a mean age of } 18 \text { years or older. } \\
\text { Diagnosis of (DSM-V) OUD. } \\
\text { Inpatients and outpatients. } \\
\text { Diagnosis of OUD with mental health disorder } \\
\text { comorbidities. } \\
\text { Diagnosis of (DSM-IV or prior) opioid dependence or } \\
\text { addiction. }\end{array}$ & $\begin{array}{l}\text { Patients younger than } 18 \text { years of age. } \\
\text { No clear diagnosis of OUD. } \\
\text { Patients mandated to treatment (ie, civil } \\
\text { commitment, drug court, diversion programme). } \\
\text { Opioid dependence in cancer pain. }\end{array}$ \\
\hline Interventions & $\begin{array}{l}\text { SDM (explicitly). } \\
\text { Patient preferences. } \\
\text { - Patient engagement. }\end{array}$ & - Interventions that do not intend to treat OUD. \\
\hline Control & Studies with or without control groups. & None. \\
\hline $\begin{array}{l}\text { Study type/ } \\
\text { design }\end{array}$ & $\begin{array}{l}\text { Peer-reviewed literature } \\
\text { Experimental studies (eg, randomised controlled trials). } \\
\text { Quasi-experimental studies (eg, pretest/post-test). } \\
\text { Observational studies (cross-sectional surveys, cohort, } \\
\text { case-control). } \\
\text { Quantitative and qualitative studies. } \\
\text { Systematic reviews. } \\
\text { Meta-analyses. }\end{array}$ & $\begin{array}{l}\text { Editorials. } \\
\text { Animal studies. } \\
\text { Case studies, case series. } \\
\text { Non-peer-reviewed literature. }\end{array}$ \\
\hline
\end{tabular}

DSM-V, Fifth Edition of the Diagnostic and Statistics Manual; OUD, opioid use disorder; SDM, shared decision-making.

(cross-sectional surveys, case-control, and prospective and retrospective cohort) and qualitative studies. Observational studies were included for review because this type of design is commonly used to investigate research questions related to patient treatment preferences. Two authors will then independently screen the remaining full-text articles. If there are disagreements regarding the included number of studies from the independent assessment, a third and neutral party will be consulted to reach an agreement.

\section{Exclusion criteria}

Case reports and editorials will be excluded. Studies whose participants were mandated to treatment (eg, through civil commitment, a diversion programme) will be excluded due to inability to provide consent or participate in SDM autonomously. Studies that are excluded will be listed with the corresponding reasons in a Preferred Reporting Items for Systematic Reviews and Meta-Analyses (PRISMA) flow diagram. ${ }^{47}$

\section{Stage 4: data extraction}

Extracting data in scoping reviews is typically an iterative and ongoing process. ${ }^{40} 42$ If new outcomes or relevant information are discovered during the course of the review, this protocol may be updated to accommodate the findings. To manage this, we developed an a priori extraction instrument to retrieve pertinent characteristics of the included studies, which will include but are not limited to: study characteristics, study objectives, participant characteristics, methods, results, the conclusion and study limitations (table 2).

We will explore and describe various health and patient-centred outcomes similarly to Shay and Lafata. ${ }^{4}$ Outcomes include, but are not limited to: changes in mental health (eg, cognitive changes, anxiety symptoms), sociobehavioural (eg, quality of life, patient satisfaction, well-being, treatment adherence) and physical health (eg, changes in weight, cardiovascular function). In order to improve the quality of the review, we will conduct a preliminary data extraction exercise and team consultation as recommended by Daudt et $a l^{41} \mathrm{We}$ will independently extract data from five randomly selected studies. The two reviewers will then meet to discuss how the data extraction instrument may need to be amended, based on its compatibility with the components of reviewed studies. If a disagreement arises, then a third reviewer with expert-level experience will be consulted for reaching final consensus. ${ }^{42}$

Many scoping reviews do not perform quality assessment of included studies. ${ }^{4043}$ However, more recently, some experts have argued that appraising the quality of included studies strengthens scoping reviews and may improve clinical or policy relevance. ${ }^{41}$ We agree with this approach, and believe assessing the quality of included studies will improve interpretability of the results. Furthermore, since we expect to include studies of various types, we will assess the quality of the included studies using the Mixed Methods Appraisal Tool. ${ }^{48}$ Two reviewers will 
Table 2 Data extraction framework

\begin{tabular}{lll}
\hline Bibliometrics & Characteristics of the review & Coding the characteristics \\
\hline Ref ID & Objective(s). & N studies that use SDM in OUD \\
First author & Study design/type. & treatment. \\
Extractor initials & Setting. & p studies of treatment preferences of \\
Year of publication & N study participants. & patients with OUD (list). \\
Country & Mean age of participants. & patients with OUD (list). \\
& Diagnosis of OUD (yes/no). & Results of included studies \\
& - Treater (N\% male). & Effect size. \\
& Control group. & Adverse events. \\
& Outcomes. & Conclusions of included studies. \\
& Was SDM evaluated in study (yes/no). & Limitations of included studies. \\
& Was patient engagement evaluated in study? (yes/no) & \\
& Were treatment preferences evaluated in study? (yes/no) & \\
\hline
\end{tabular}

OUD, opioid use disorder; SDM, shared decision-making.

independently perform the quality assessment, and a third reviewer will resolve any disagreements.

\section{Stage 5: data summary and synthesis of the results}

Scoping reviews aim to summarise a wide range of findings rather than provide a quantitative synthesis that is typically seen in systematic reviews. ${ }^{40-43}$ Subsequently, this review will provide a descriptive summary of the included peer-reviewed articles. We anticipate that some analyses will be quantitative in nature while others will be qualitative. Any additional findings or trends in the data relating to the decision-making process in OUD treatment will be discussed. We will identify gaps in knowledge and make recommendations for future research, practice and policy, keeping in mind the needs of our stakeholders. The PRISMA-Protocols reporting guidelines for systematic reviews will be followed to ensure rigour when reporting the results. ${ }^{47}$

\section{Stage 6: consultation}

Consultation enhances methodological rigour in scoping reviews. ${ }^{4142}$ As a result, we will employ a two-part consultation process. First, we held preliminary consultations with policy-makers, clinicians, community health centres and researchers with relevant expertise, to promote integrated knowledge translation. Our aim was to use consultation to help develop a research question that would generate the knowledge needed to address the current opioid crisis.

Examples of consultation questions included:

1. How might SDM help ameliorate the current opioid crisis?

2. How might a scoping review on SDM in OUD treatment affect your work?

3. What are the advantages/pros and barriers/cons to implementing SDM in OUD treatment?

4. Is SDM in OUD treatment represented in the existing literature or clinical practice? If not, do you think it deserves more attention?
5. Are there any experts in the field you'd recommend speaking with or any literature we should be sure to read?

We are currently following up responses to question 5, and aim to create a 'snowball' sample of highly knowledgeable individuals.

The second stage of the consultation process will take place after the data are extracted. Initial results from 'stage 5' will be discussed with the aforementioned stakeholders. At this time, they will be given another opportunity to provide feedback, suggest more experts and recommend additional literature. Once the review is complete, the experts and stakeholders will be contacted again to discuss practice and policy implications from the results.

\section{Patient and public involvement}

Patients were not involved during the development of this scoping review protocol. An initial draft of the final results will be prepared and sent to various primary care and mental health clinics part of a larger 'parent' SDM initiative, to be circulated to staff for feedback. We will collaborate with each site to obtain feedback on the initial draft of a summary of the final results from two to three patients/clients at each site. We will then revise the draft of the final results and will create a lay summary as needed. A summary of our review, with any changes suggested by staff or patients/clients incorporated, will be placed on the University of Alberta Integrative Health Institute website.

\section{ETHICS AND DISSEMINATION}

The purposes of this scoping review are: (1) conduct a broad search for literature related to the impact of SDM for the treatment of OUD in adults and (2) descriptively summarise the results of the literature. This scoping review will employ a high level of methodological rigour in accordance with the scoping review frameworks of Arksey and O'Malley ${ }^{40}$ and Levac et $a l^{42}$ A comprehensive search 
strategy using five databases and broad search terms will be employed to retrieve a large body of peer-reviewed literature. Due to the breadth and likely heterogeneity of the literature, data extraction will be an iterative process, and the synthesis will provide an overview and map any concepts that are identified. The results from this review will highlight gaps in knowledge, establish new possibilities and identify potential barriers and facilitatorsto treatment outcomes for adult patients with OUD. The final manuscript will be submitted to a peer-reviewed journal, disseminated at relevant academic conferences and circulated to policy-makers and to our clinical stakeholders.

\section{Author affiliations}

${ }^{1}$ Department of Psychiatry, Faculty of Medicine \& Dentistry, University of Alberta, Edmonton, Alberta, Canada

${ }^{2}$ Department of Epidemiology, University of California Berkeley School of Public Health, Berkeley, California, United States of America

${ }^{3}$ School of Public Health, University of Alberta, Edmonton, Alberta, Canada ${ }^{4}$ Department of Pediatrics, Faculty of Medicine \& Dentistry, University of Alberta, Edmonton, Alberta, Canada

${ }^{5}$ Faculty of Nursing, University of Alberta, Edmonton, Alberta, Canada

${ }^{6}$ Addiction and Mental Health Strategic Clinical Network, Alberta Health Services, Edmonton, Alberta, Canada

${ }^{7}$ Department of Medicine, Faculty of Medicine \& Dentistry, University of Alberta, Edmonton, Alberta, Canada

Acknowledgements We would like to thank our community stakeholders, contributors, and reviewers for their input and feedback towards this work.

Contributors All authors have made significant intellectual contribution to the development of this protocol. TM and ENK familiarised themselves with the extant scoping review literature. TM developed the research questions, produced the first draft of the manuscript. ENK, SK-J, KO and MH assisted in development of the research questions and revisions of the manuscript. SK-J designed the search strategy and performed the database searches. ENK consulted public health subject matter experts in the USA. AA-A and KR provided substantial clinical expertise and edited the manuscript. SV has expertise in conducting literature reviews, and provided detailed feedback on all protocol drafts. All authors have approved the final protocol before publication.

Funding The authors have not declared a specific grant for this research from any funding agency in the public, commercial or not-for-profit sectors.

Competing interests None declared.

Patient consent Not required.

Ethics approval Since this is a literature review, and no human participants were contacted during the development of this study, ethics approval is not required.

Provenance and peer review Not commissioned; externally peer reviewed.

Open access This is an open access article distributed in accordance with the Creative Commons Attribution Non Commercial (CC BY-NC 4.0) license, which permits others to distribute, remix, adapt, build upon this work non-commercially, and license their derivative works on different terms, provided the original work is properly cited, appropriate credit is given, any changes made indicated, and the use is non-commercial. See: http://creativecommons.org/licenses/by-nc/4.0/.

\section{REFERENCES}

1. Barry MJ, Edgman-Levitan S. Shared decision making-pinnacle of patient-centered care. N Engl J Med 2012;366:780-1.

2. Elwyn G, Frosch D, Thomson R, et al. Shared decision making: a model for clinical practice. J Gen Intern Med 2012;27:1361-7.

3. Luty J. Treatment preferences of opiate-dependent patients. The Psychiatrist 2004;28:47.

4. Shay LA, Lafata JE. Where is the evidence? A systematic review of shared decision making and patient outcomes. Med Decis Making 2015;35:114-31.
5. Montori VM, Gafni A, Charles C. A shared treatment decisionmaking approach between patients with chronic conditions and their clinicians: the case of diabetes. Health Expect 2006;9:25-36.

6. Friedrichs A, Spies M, Härter M, et al. Patient preferences and shared decision making in the treatment of substance use disorders: a systematic review of the literature. PLoS One 2016;11:e0145817.

7. Busse JW, Juurlink D, Guyatt GH. Addressing the limitations of the CDC guideline for prescribing opioids for chronic noncancer pain. CMAJ 2016;188:1210-1.

8. Health C. Taking action on canada's opioid crisis 2017.

9. Rudd RA, Aleshire N, Zibbell JE, et al. Increases in drug and opioid overdose deaths-United States, 2000-2014. MMWR Morb Mortal Wkly Rep 2016;64:1378-82.

10. Slavova S, O'Brien DB, Creppage K, et al. Drug overdose deaths: let's get specific. Public Health Rep 2015;130:339-42.

11. Suzuki J, El-Haddad S. A review: fentanyl and non-pharmaceutical fentanyls. Drug Alcohol Depend 2017;171:107-16.

12. Dowell $D$, Noonan RK, Houry D. Underlying factors in drug overdose deaths. JAMA 2017:318:2295-6.

13. McCall Jones C, Baldwin GT, Compton WM. Recent increases in cocaine-related overdose deaths and the role of opioids. Am J Public Health 2017:107:430-2.

14. Klar SA, Brodkin E, Gibson E, et al. Notes from the field: furanylfentanyl overdose events caused by smoking contaminated crack cocaine - British Columbia, Canada, July 15-18, 2016. MMWR Morb Mortal Wkly Rep 2016;65:1015-6.

15. American Psychiatric Association. Diagnostic and statistic manual of mental disorders. 5th edn, 2013.

16. Hall W, Carter A, Forlini C. The brain disease model of addiction: is it supported by the evidence and has it delivered on its promises? Lancet Psychiatry 2015;2:105-10.

17. Patel RS, Elmaadawi A, Nasr S, et al. Comorbid post-traumatic stress disorder and opioid dependence. Cureus 2017;9:e1647.

18. Center of Disease Control. Wide-ranging online data for epidemiologic research (WONDER). Atlanta: Center of Disease Control, 2016.

19. Mannelli P, Wu LT, Lt W. Primary care for opioid use disorder. Subst Abuse Rehabil 2016;7:107-9.

20. Redko C, Rapp RC, Carlson RG. Waiting time as a barrier to treatment entry: perceptions of substance users. J Drug Issues 2006;36:831-52.

21. Samet JH, Fiellin DA. Opioid substitution therapy-time to replace the term. Lancet 2015;385:1508-9.

22. Day E, Strang J. Outpatient versus inpatient opioid detoxification: a randomized controlled trial. J Subst Abuse Treat 2011;40:56-66.

23. Winstanley EL, Brigham GS, Babcock D, et al. Improving treatment for opioid dependence: a perspective from the Ohio Valley node of the NIDA clinical trials network. Prog Community Health Partnersh 2014;8:99-107.

24. Dick AW, Pacula RL, Gordon AJ, et al. Increasing potential access to opioid agonist treatment in U S. treatment shortage areas. Health Affairs 2015;34:1028-34.

25. Andrilla CHA, Moore TE, Patterson DG, et al. Geographic distribution of providers with a DEA waiver to prescribe buprenorphine for the treatment of opioid use disorder: a 5-year update. J Rural Health 2018.

26. APSE, 2017. Continuing progress on the opioid epidemic: the role of the affordable care act. https://aspe.hhs.gov/pdf-report/continuingprogress-opioid-epidemic-role-affordable-care-act

27. Smyth BP, Barry J, Keenan E, et al. Lapse and relapse following inpatient treatment of opiate dependence. Ir Med J 2010;103:176-9.

28. Sharma B, Bruner A, Barnett G, et al. Opioid use disorders. Child Adolesc Psychiatr Clin N Am 2016;25:473-87.

29. Bruneau J, Ahamad K, Goyer MĖ, et al. Management of opioid use disorders: a national clinical practice guideline. CMAJ 2018;190:E247-57

30. Strang J, McCambridge J, Best D, et al. Loss of tolerance and overdose mortality after inpatient opiate detoxification: follow up study. BMJ 2003;326:959-60.

31. Schwartz RP, Kelly SM, O'Grady KE, et al. Attitudes toward buprenorphine and methadone among opioid-dependent individuals. Am J Addict 2008;17:396.

32. Meier PS, Barrowclough C, Donmall MC. The role of the therapeutic alliance in the treatment of substance misuse: a critical review of the literature. Addiction 2005;100:304-16.

33. Joosten EA, De Weert-Van Oene GH, Sensky T, et al. Treatment goals in addiction healthcare: the perspectives of patients and clinicians. Int J Soc Psychiatry 2011;57:263-76.

34. Nelson LS, Juurlink DN, Perrone J. Addressing the opioid epidemic. JAMA 2015;314:1453-4. 
35. Davis C, Green T, Beletsky L. Action, not rhetoric, needed to reverse the opioid overdose epidemic. J Law Med Ethics 2017;45(Suppl 1):20-3.

36. Volkow ND, Collins FS. The role of science in addressing the opioid crisis. N Engl J Med 2017;377:391-4.

37. Dasgupta N, Beletsky L, Ciccarone D. Opioid crisis: no easy fix to its social and economic determinants. Am J Public Health 2018;108:182-6.

38. O'Brien C. Addiction and dependence in DSM-V. Addiction 2011:106:866-7.

39. Hasin DS, O'Brien CP, Auriacombe M, et al. DSM-5 criteria for substance use disorders: recommendations and rationale. $A m \mathrm{~J}$ Psychiatry 2013;170:834-51.

40. Arksey H, O'Malley L. Scoping studies: towards a methodological framework. Int J Soc Res Methodol 2005;8:19-32.

41. Daudt HM, van Mossel C, Scott SJ. Enhancing the scoping study methodology: a large, inter-professional team's experience with Arksey and O'Malley's framework. BMC Med Res Methodol 2013;13:48.

42. Levac D, Colquhoun H, O'Brien KK. Scoping studies: advancing the methodology. Implement Sci 2010;5:69.
43. Khalil H, Peters M, Godfrey CM, et al. An evidence-based approach to scoping reviews. Worldviews Evid Based Nurs 2016;13:118-23.

44. Joosten EA, de Jong CA, de Weert-van Oene GH, et al. Shared decision-making reduces drug use and psychiatric severity in substance-dependent patients. Psychother Psychosom 2009;78:245-53.

45. Katz AL, Webb SA. Committee on Bioethics. Informed consent in decision-making in pediatric practice. Pediatrics 2016;138:e20161485.

46. Daly RL, Bunn F, Goodman C. Shared decision-making for people living with dementia in extended care settings: a systematic review. BMJ Open 2018;8:e018977.

47. Moher D, Shamseer L, Clarke M, et al. Preferred reporting items for systematic review and meta-analysis protocols (PRISMA-P) 2015 statement. Syst Rev 2015;4:1):1.

48. Pace R, Pluye P, Bartlett G, et al. Testing the reliability and efficiency of the pilot Mixed Methods Appraisal Tool (MMAT) for systematic mixed studies review. Int J Nurs Stud 2012;49:47-53. 